\title{
Energy Modeling and Adaptive Sampling Algorithms for Energy Harvesting Powered Nodes with Sampling Rate Limitations
}

\author{
Elvina Gindullina ${ }^{1}$ | Leonardo Badia ${ }^{1}$ | \\ Xavier Vilajosana ${ }^{2}$
}

\footnotetext{
${ }^{1}$ Department of Information Engineering, University of Padova, Padova, PD, 35131 Italy

${ }^{2}$ Computer Science, Telecommunications and Multimedia Department, Open University of Catalonia, Barcelona, Catalunya, 08018 Spain

Correspondence

Elvina Gindullina, Department of Information Engineering, University of Padova, Padova, PD, 35131 Italy Email: elvina.gindullina@dei.unipd.it

\section{Funding information}

This work has received funding from the European Union's Horizon 2020 research and innovation programme under the Marie Sklodowska-Curie grant agreement No. 675891 (SCAVENGE)
}

This article explores the implementation of different sampling strategies for a practical energy harvesting wireless device (sensor node) powered by a rechargeable battery. We look for a realistic yet effective sampling strategy that prevents packet delivery failures, which is simple enough to be implemented in low complexity hardware. The article proposes methods that balance erratic energy arrivals and include advantages of dynamic data-driven approaches based on historical data. Due to the industrial requirements in terms of minimum acceptable sampling frequency, we also integrate sampling rate limits and verify the proposed methods. To do so, we simulated the operation of an industrial data-logger powered with a solar panel relying on the enhanced state of the model for battery charging. Finally, the proposed methods are compared based on energy consumption over a year and amount of packet delivery failures, thus showing how some modifications of available strategies achieve satisfactory performance in this sense.

KEYWORDS

adaptive sampling algorithm, energy harvesting, Internet of Things, sampling rate, self-sustainability, solar energy, state of the charge. 


\section{1 | INTRODUCTION}

Industrial wireless data-loggers are usually deployed in remote or outdoor areas and are mainly powered by batteries with limited capacity. It is an actual issue for vendors to provide guarantees in terms of the overall operational time, considering different sampling possibilities.

A widely used approach to increase the autonomy of the devices is the usage of renewable sources of energy. However, such sources are too erratic to provide complete system reliability unless over-dimensioned. In reality, energy supply is often limited, which causes the need for adaption of the node operational strategy to ensure the functional reliability of the system.

Solar, wind, heat, and other renewable sources of energy can be used to power the devices. One of the most widespread solutions for wireless sensor nodes is the use of solar panels, which can provide reasonable power input. Their efficiency is determined by the panel's material that defines the conversion efficiency [1].

One of the possible ways to adapt the energy consumption of a wireless sensor device to a harvesting pattern and build an energy-sustainable system is to adjust its sampling rate. The sampling frequency or sampling rate is the average number of samples collected in one second. Sampling rate significantly affects the energy consumption of a sensing device. If a device goes out of charge, then it fails to deliver a data packet. This might be even more significant if data-driven sampling approaches are adopted. This happens, for example, when a sample is gathered if the difference in data values are high enough, and/or a packet failed delivery, so that a gap is present in the collected data.

However, the erratic nature of the ambient energy requires to adopt a sampling strategy that, on one hand, tailors the sampling rate to the underlying energy arrival process, while on the other hand being implementable on simple hardware. Therefore, we seek a strategy that takes advantage of data-driven approaches, is readily implementable to the state-of-the-art devices, and balances volatile energy arrivals.

For these reasons, this work investigates possible extensions to improve the performance of the data-driven adaptive sampling algorithm (DDASA) [2] in terms of energy awareness, taking advantage of other ideas presented in the literature. Also, we complement the algorithm with sampling rate limitations, regarded as constraints to the adaptive sampling policy, which are realistically present in industrial applications.

The proposed algorithms aim at balancing the performance of the sensor device considering energy harvesting capabilities as well as its current battery status. We compared the proposed solutions with DDASA and a strategy with constant sampling rate and energy arrivals.

To perform a realistic assessment of the resulting performance, we tried to realistically simulate all operational aspects, including an accurate model of the environment, energy harvesting, and battery behaviors, so as to derive a correct quantification of the state of charge (SoC) as well as the energy consumption of the device. As we found out, most of the evaluations in the literature do not take all these aspects into account. For instance, most of the SoC models do not consider battery deterioration due to continuous usage or environmental factors.

Therefore, we considered an extension of those models to a practical setup to derive a realistic SoC estimation. For our validation, we used a LoadSensing commercial data-logger (further - LS) [3] powered by the commercial solar panel SOLEM 10/150/100 TD. We forecast the operation of the industrial data-logger for a period of one year and compared the performance under different sampling rate strategies.

The article is organized as follows. The state of the art and background information in adaptive sampling for wireless sensing devices is given in Section 2. In Section 3, we introduce our sampling policies dealing with sampling rate limitations. In the following section, we present the simulator system model (Section 4). In particular, the solar irradiation model is discussed in subsection 4.1, the photovoltaic (PV) power output model is presented in subsection 4.2, the improvements of SoC estimation is demonstrated in subsection 4.3. Numerical results are discussed in Section 
5. Finally, conclusions are outlined in Section 6.

\section{2 | BACKGROUND IN ADAPTIVE SAMPLING FOR SENSING DEVICES}

According to [4], energy management in WSN is defined as a set of instructions to efficiently handle power consumption and energy provision in a constrained sensor node. In the literature, papers dealing with energy management try to either enhance their provisioning, or minimize the energy consumption.

For the latter goal, i.e., to reduce (or adapt) the energy consumption, duty-cycling, data-driven and mobility based approaches are considered. Duty cycles is one of the most effective way to improve the network energy sustainability. In [5], the authors propose to adjust the nodes duty cycles, or, in other words, the wake/sleep phases. The volatility of the energy arrivals is accounted by energy prediction. The authors of [6] proposed a method to reduce the energy consumption by adjusting the sensing duty cycles according to the available energy levels. Mobility based approaches consider the mobile nodes in the network [4], [7].

Finally, data-driven approaches are based on spatial correlation of data, and aim to reduce the amount of the sampled data keeping the sensing accuracy within an acceptable range. These approaches are subdivided on data prediction schemes and data acquisition approaches. Data acquisition schemes try to reduce the energy consumption in the node sensing subsystems, and can be implemented using three different approaches [4]: hierarchical sensing, adaptive sampling, and model-based active sensing. In hierarchical sensing, multiple sensors are installed on the sensor nodes and observe the same event with a different resolution and power consumption. Hierarchical sensing can be divided into two types:

- triggered sensing - when more accurate and power-consuming sensors are activated after the low-resolution sensors to detect some activity within the sensed area;

- multi-scale sensing - identifies areas within a region that require more accurate monitoring.

Adaptive sampling techniques provide online sampling frequencies for sensing nodes and change the sampling rate by evaluating correlations between the sensed data and the available energy [8]. If the subsequent samples do not differ very much, then it is possible to reduce the sampling rate based on this temporal correlation. Another possibility to decrease the overall energy consumption by adapting the sampling rate frequency is to apply harvesting aware optimization of the power consumption using the known remaining battery level and forecast harvested energy.

Model-based active sensing is a forecasting model of the sensing phenomena based on an initial set of sampled data. As underlined in [9], some sensors may even consume significantly more energy than the transmission component. The authors propose a general approach that leverages two complementary mechanisms at the sensor level: 1) duty cycling (i.e., the sensor board is switched off between two consecutive samples) and 2) adaptive sampling (i.e., the optimal sampling frequency is estimated on-line). The proposed adaptive sampling algorithm (ASA) in [9] is tested on a snow-monitoring applications sensor. It is demonstrated that ASA performs $79 \%$ more efficiently in terms of energy consumption in comparison with the constant sampling rate. Nevertheless, the algorithm has room for enhancement regarding the residual energy level.

Srbinovski et al. [10] introduce the energy aware adaptive sampling algorithm (EASA), that modifies the ASA algorithm by taking into account the current energy level of a sensor. That is, ASA is combined with an energy aware function, assuming that each node in the network is able to monitor its own energy level. The sampling rate of EASA is consecutively decreased under certain energy level without limitation since the sampling rate of EASA is proportional to the remaining energy of nodes. EASA is evaluated on two testbeds powered by two sources of energy - wind and 
solar, and is demonstrated that EASA outperforms ASA.

Other energy-aware adaptive sampling algorithms are proposed in [11]: Resuscitation Adaptive Sampling Algorithm (RASA) and Compensation Adaptive Sampling Algorithm (CASA). The purpose of RASA is to set low sampling rate and guarantee self-sustainability when energy state of sensors is extremely low. Sensor nodes in CASA can be recharged by saving the consumption energy when the harvesting quality is good. The algorithms are compared with ASA and demonstrated a better performance in terms of energy consumption.

An optimal scheduling sensing policy for an energy harvesting system equipped with a finite battery is considered in [12]. The objective is to select the sensing epochs such that the long-term average sensing performance is optimized. Finding the optimal solution can be a computationally intensive task and requires a device to have sufficient computational capabilities.

The data-driven approach is adopted to develop ASA for power management in automated monitoring of the quality of water. Data-driven adaptive sampling algorithm (DDASA) is proposed in [2]. DDASA can save $30.66 \%$ of energy for three months in comparison with the fixed rate strategy. DDASA changes the sampling frequency based on the nature of the sampled ratio compared with ASA. A sigmoid function is proposed to dynamically set the sampling frequency. DDASA is tested on a device, powered by a non-rechargeable battery, thus, it does not take into account the harvesting capabilities as well as battery level.

From the described algorithms only CASA takes into account energy harvesting potential of a sensing device, even though the benefits of using a data-driven approach such as including the data accuracy in the optimization are not incorporated. Therefore, one of the objective of this work is to adjust the data driven approach to calculate the sampling rate of a battery and harvester equipped device. Yet, we integrate sampling rate limitations as a mechanism to adapt to the industrial requirements.

In the literature, further adaptive sampling algorithms for more specific applications in WSN are proposed. In particular, in [13] optimality criteria for mobile robotic wireless sensor network is suggested to the most informative location of interest. The adaptive sampling strategy for mobile sensors in the environment monitoring context was proposed in [14], where the sequential Bayesian prediction algorithm minimizes the prediction error variance. An adaptive sampling system for sensor network is considered in [15], that is, the analog method for signal dependent ADC clocking. Another adaptive sampling algorithm was proposed in [16] for target tracking in underwater wireless sensor networks, that simultaneously balance the energy consumption and maximizes the energy efficiency. All of these proposals are specific to their applications and leverage some further aspects of their scenarios. Even though we considered a definite use case related to the geotechnical industry, our proposal is instead more general and we believe that we can extend our same rationale to all these contributions to improve their results.

\section{3 | ADAPTIVE SAMPLING ALGORITHMS WITH SAMPLING RATE LIMITATIONS}

Adaptive sampling algorithms estimate at runtime the expedient sampling frequencies for sensor devices.

Sampling algorithms can be extended by including sensing frequency limitations. This is motivated by the industrial requirement of guaranteeing a certain amount of data per unit of time. In particular, it might be desirable to limit the minimum sensing frequency, while maximum sampling rate can be as high as possible. In this case, the maximum frequency can be only bound by a duration of a cycle, in which a sensor performs warming up, measurement and transmissions:

$$
F_{\max }[H z]=\frac{1}{t_{\text {warm }}+t_{\text {meas }}+t_{\text {trans }}},
$$




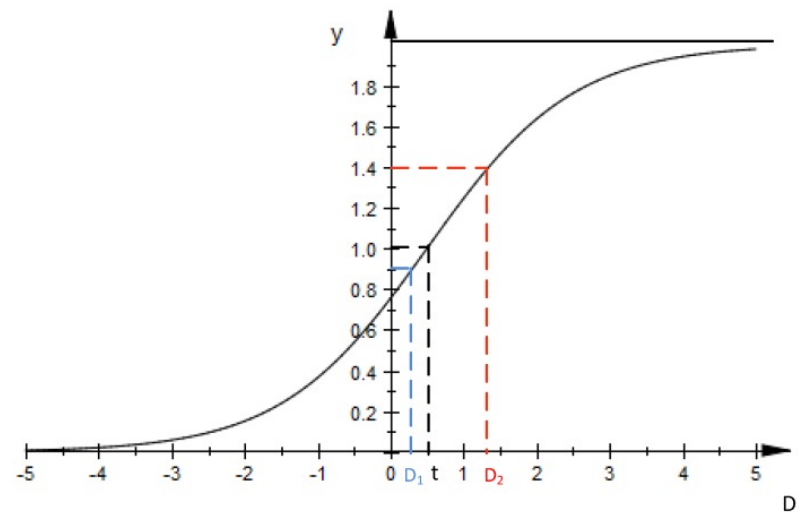

FIGURE 1 Revised sigmoid function [2]

where $t_{\text {warm }}, t_{\text {meas }}$ and $t_{\text {trans }}$ are the time required for warming up of the sensor, taking a measurement and transmitting the measurement, respectively.

In this section, we propose 4 methods taking into account energy harvesting capabilities and battery level information, in order to improve the DDASA performance. Differently from DDASA, the proposed methods include sampling rate limitations, energy capabilities as well as the capability to sectorize the battery level and apply different rules to the different sectors.

Sampling rate limits $F_{\max }$ and $F_{\min }$ serve as boundaries for the proposed adaptive sampling algorithms.

The original DDASA changes the sampling frequency based on the nature of sampled data (Algorithm 1). Specifically, DDASA updates the sampling frequency based on the sigmoid function $y(D)(0<y(D)<2)$, where $D$ is calculated as a difference between two measurements $x_{i}$ and $x_{i+1}$ over the average value of the $N$ recent data. $D$ rises if the environment suddenly changes (Fig. 1).

The sigmoid function represents a deterministic growth pattern. The simple way to represent the sigmoid function is [17]:

$$
w=\frac{w_{\max }}{1+e^{-k\left(t-t_{m}\right)}},
$$

where $w$ is the weight to be calculated, $w_{\max }$ is the maximum value of $w, t_{m}$ is the period of time when the maximum value of $w$ was observed, and $k$ defines the curvature of the pattern.

Our first proposal is threshold-based ASA or T-ASA, which is based on the energy level and harvesting rate thresholds, and corrects the sampling rate when the energy level or energy arrival rate go beyond a threshold. T-ASA utilizes the mechanisms proposed in [10] and [11]. Based on their approach, we propose the mapping between different battery and energy arrival levels (thresholds) and equations that adjust the sampling rate. This method considers four states:

1. High energy arrivals $\left(H / H_{\max }>k\right)$ and high battery level $\left(E_{\text {batt }}>E_{\text {th }}\right): f_{\text {new }}=f_{\text {curr }}$;

2. High energy arrivals $\left(H / H_{\max }>k\right)$ and low battery level $\left(E_{\text {batt }}<E_{\text {th }}\right): f_{\text {new }}=f_{\text {curr }} \cdot\left(\frac{E_{t h}-E_{\text {batt }}}{100}\right)^{m}$;

3. Low energy arrivals $\left(H / H_{\max }<k\right)$ and high battery level $\left(E_{\text {batt }}>E_{\text {th }}\right): f_{\text {new }}=f_{\text {curr }} \cdot\left(1+\frac{H}{H_{\max }}\right) \cdot N$;

4. Low energy arrivals $\left(H / H_{\max }<k\right)$ and low battery level $\left(\left(E_{\text {batt }}<E_{\text {th }}\right): f_{\text {new }}=f_{\text {curr }} \cdot\left(\frac{E_{t h}-E_{\text {batt }}}{100}\right)^{m} \cdot\left(1+\frac{H}{H_{\max }}\right) \cdot N\right.$,

Parameter $k$ is an energy arrivals threshold, $m$ and $N$ denote the parameters of the algorithm, $H$ and $H_{\max }$ are current solar radiation and maximum possible solar energy arrival, correspondingly, $E_{b a t t}$ and $E_{t h}$ are the current 


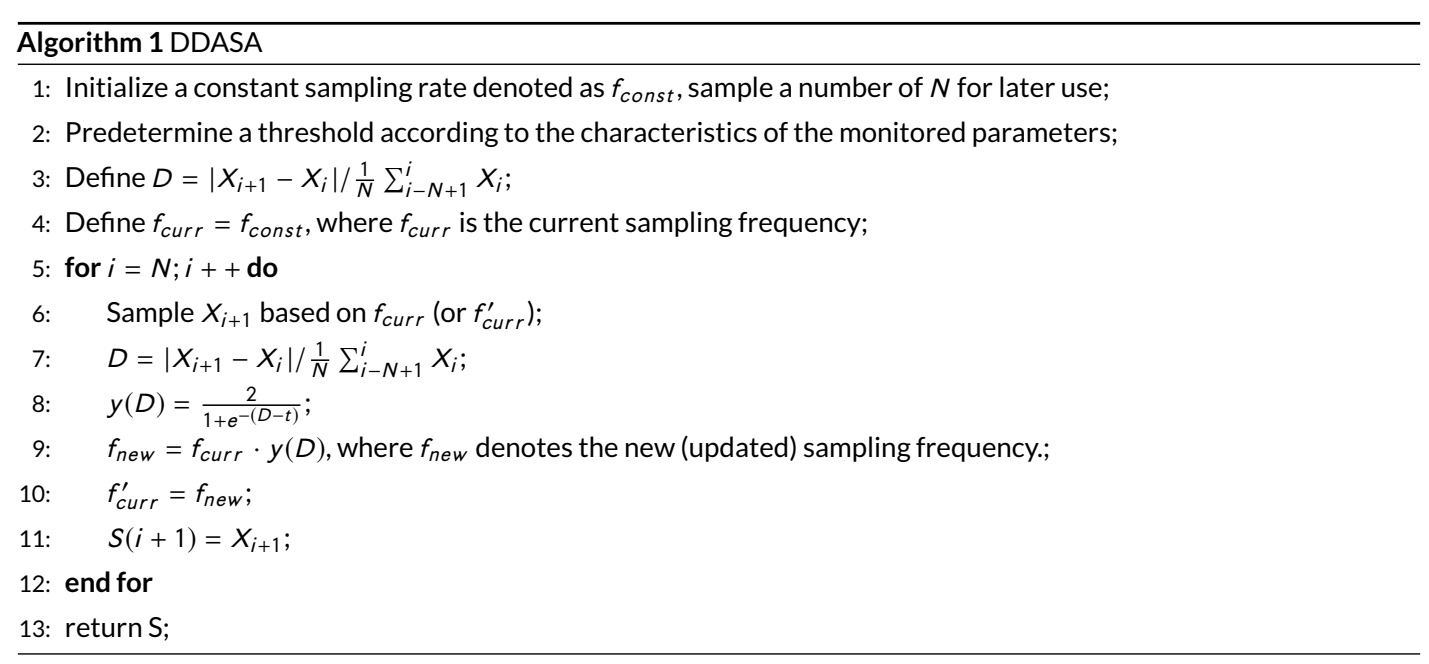

battery level (\%) and battery threshold (\%). Coefficient $\left(E_{t h}-E_{\text {batt }}\right) / 100 \in[0,1]$ represents the deviation of the energy level from its threshold. The parameter $m$ adjusts the granularity of the algorithm. Higher values of $m$ decrease the value of the sampling frequency more significant. In other words, $m$ is adjusted depending on the strength of the required intervention. Coefficient $\left(1+H / H_{\max }\right) \in[1,2]$ increases the value of the sampling frequency in the case of more frequent energy arrivals. Parameter $N \in(0,1]$ similarly with $m$ defines the granularity of the method.

The second method, analogously to T-ASA, uses $f_{\max }$ and thresholds. However, instead of correcting the current sampling rate as done in the previous method, the calculation is based on the sampling rate limit $f_{\max }$ and current energy capabilities. The method is defined as L-ASA, or limits-based ASA:

1. High energy arrivals $\left(H / H_{\max }>k\right)$ and high battery level $\left(E_{\text {batt }}>E_{t h}\right): f_{\text {new }}=f_{\text {max }}$;

2. High energy arrivals $\left(H / H_{\max }>k\right)$ and low battery level $\left(E_{\text {batt }}<E_{t h}\right): f_{\text {new }}=f_{\max } \cdot\left(\frac{E_{t h}-E_{\text {batt }}}{100}\right)^{m}$;

3. Low energy arrivals $\left(H / H_{\max }<k\right)$ and high battery level $\left(E_{\text {batt }}>E_{t h}\right): f_{\text {new }}=f_{\max } \cdot\left(1+\frac{H}{H_{\max }}\right) \cdot N$;

4. Low energy arrivals $\left(H / H_{\max }<k\right)$ and low battery level $\left(\left(E_{\text {batt }}<E_{\text {th }}\right): f_{\text {new }}=f_{\max } \cdot\left(\frac{E_{t h}-E_{\text {batt }}}{100}\right)^{m} \cdot\left(1+\frac{H}{H_{\max }}\right) \cdot N\right.$,

In the third method called LT-DDASA (limits and thresholds based DDASA), we propose to adjust the sampling rate to its limits if the following conditions are satisfied:

1. High battery level $\left(E_{\text {batt }}>E_{t h}^{u p}\right): f_{\text {new }}=f_{\text {max }}$;

2. Low battery level $\left(E_{\text {batt }}>E_{\text {th }}^{\text {low }}\right): f_{\text {new }}=f_{\text {min }}$

If $E_{t h}^{\text {low }}<E_{\text {bat }}<E_{t h}^{\text {up }}$ then sampling rate is determined by DDASA. To take into account the harvesting capabilities of a sensor node, we introduce the calculation of derivatives, that determines the period of time when the energy arrivals have a tendency to grow or decrease over time.

3. if $\frac{d f}{d t}>0: f_{\text {new }}=f_{\text {curr }} * \alpha$, where $\alpha(0<\alpha \leq 1)$ is a coefficient increasing the sampling frequency;

4. if $\frac{d f}{d t}<0: f_{\text {new }}=f_{\text {curr }} * \beta$, where $\beta(0<\beta \leq 1)$ is a coefficient decreasing the sampling frequency. 
Finally, EA-DDASA (energy-aware DDASA) is based on the calculation of the sigmoid function presented in DDASA. In contrast with DDASA, we include the calculation of the sigmoid function not only for collected data, but also for energy arrivals and battery level:

$$
\begin{array}{r}
y(D)=\frac{2}{1+e^{-(D-t)}} \\
x(S \circ C)=\frac{2}{1+e^{-(S \circ C-k)}} \\
z(H)=\frac{2}{1+e^{-\left(k-\frac{H}{H}\right)}} \\
f_{\text {new }}=f_{\text {curr }} \cdot y(D) \cdot x(S \circ C) \cdot z(H)
\end{array}
$$

All three components in (3) are combined to define the value of sampling rate, so that, for instance, low values of battery level can be compensated by high energy arrivals.

In order to validate the proposed methods, we simulate the operation of an industrial sensor node powered by a solar panel. Simulations are based on the system model presented in the following section.

\section{4 | SYSTEM MODEL}

To test the proposed adaptive sampling algorithms we introduce the energy model for the energy-harvesting wireless sensor, specifically, for a tiltmeter powered by a solar panel.

To analyze the sustainability of the solar-powered sensor device with integrated adaptive sampling algorithm, we first describe our model for the node SoC. This can be divided into four stages:

1. Model of the solar irradiation taking into account meteorological conditions, location, reflection, solar panel inclination, soiling effects, etc.

2. Model of the power output based on the inner characteristics of the solar panel, such as cell temperature, area, losses, solar radiation on the tilted surface etc.

3. The actual load model based on the battery effects, such as battery degradation and duty cycling

4. The energy consumption model based on the expenditure for one sensing cycle and the adopted adaptive sampling algorithm

\subsection{Solar irradiation modeling}

Solar irradiation represents the amount of solar power (or instantaneous energy) per unit area $\left[\mathrm{W} / \mathrm{m}^{2}\right]$. Few parameters that determine the solar irradiation on the surface of Earth are discussed in [18]: the Earth's geometry and location (declination, latitude, solar hour angle); terrain (elevation, surface inclination and orientation, shadows); atmospheric attenuation (scattering, absorption) by gases, solid and liquid particles and clouds.

Different combinations of these parameters are included in the solar irradiation models. Global solar energy models are considered in [19], divided into two components: extraterrestrial and global solar energy, i.e. above or below the atmosphere, respectively. Global energy models may be further categorized into computation of direct beams and diffuse solar energy. These parameters are usually measured, but the installation of measurement devices is costly. Therefore, prediction models are widely used to measure the global solar radiation [19].

We tested and compared two models to obtain an input solar radiation, namely an astronomical model and a 


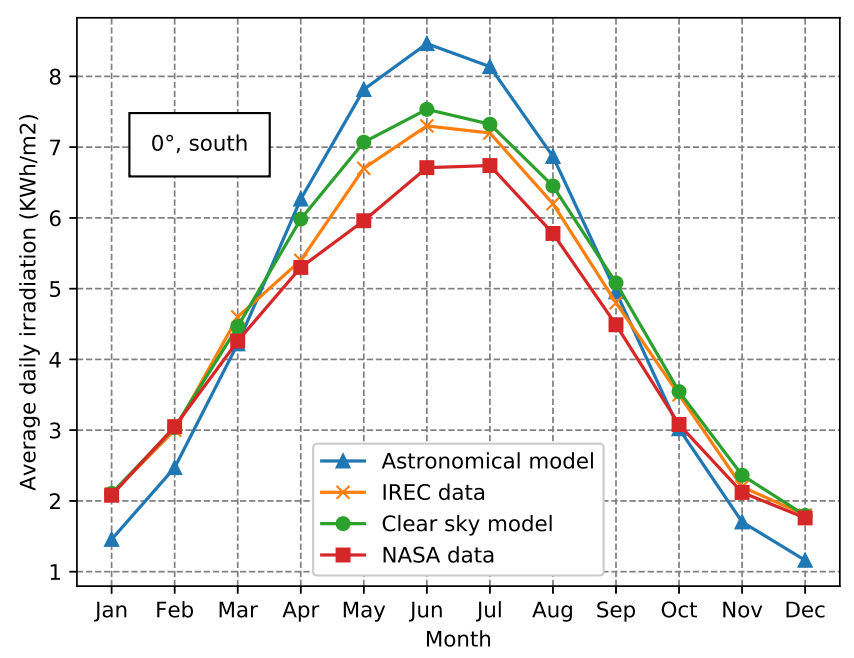

FIGURE 2 Comparison of average daily irradiation for $0^{\circ}$

clear-sky model (see description in Appendix A), that do not demand the real data sheets, although the knowledge of the reflection characteristics of the location and ground are needed. We set the reflection parameters that correspond to the concrete surroundings since we consider the urban scenario.

The models were implemented and compared with real data, provided by IREC (Institut de Recerca en Energia de Catalunya) for Barcelona, Spain, and with the database of NASA for a tilted solar panel: $0^{\circ}$ (Fig.2) , 37 (Fig.3) and $90^{\circ}$ (Fig.4). For this purpose we aggregated hourly data over one year, obtained as an output of these two models. The incident solar power data for the input of an astronomical model was derived from [20].

For the performance evaluation of the models, we consider the mean square error $E$ of the average daily irradiation $y$. That is, if $y_{i}$ is a data point and $\hat{y}_{i}$ is its estimate, we compute $E$ as:

$$
E=\sum_{i=1}^{N}\left(y_{i}-\hat{y}\right)^{2}
$$

A comparison of the results for two models is presented in Table 1. Clear-sky model showed higher accuracy in comparison with the astronomical model.

TABLE 1 Error values

\begin{tabular}{|l|c|c|c|c|c|c|}
\hline & \multicolumn{3}{|c|}{ Astronomical model } & \multicolumn{3}{c|}{ Clear sky model } \\
\hline Data source & $0^{\circ}$ & $37^{\circ}$ & $90^{\circ}$ & $0^{\circ}$ & $37^{\circ}$ & $90^{\circ}$ \\
\hline IREC & 6.421 & 7.654 & 2.462 & 0.738 & 0.871 & 1.507 \\
\hline NASA & 12.064 & - & 2.196 & 3.839 & - & 2.005 \\
\hline
\end{tabular}




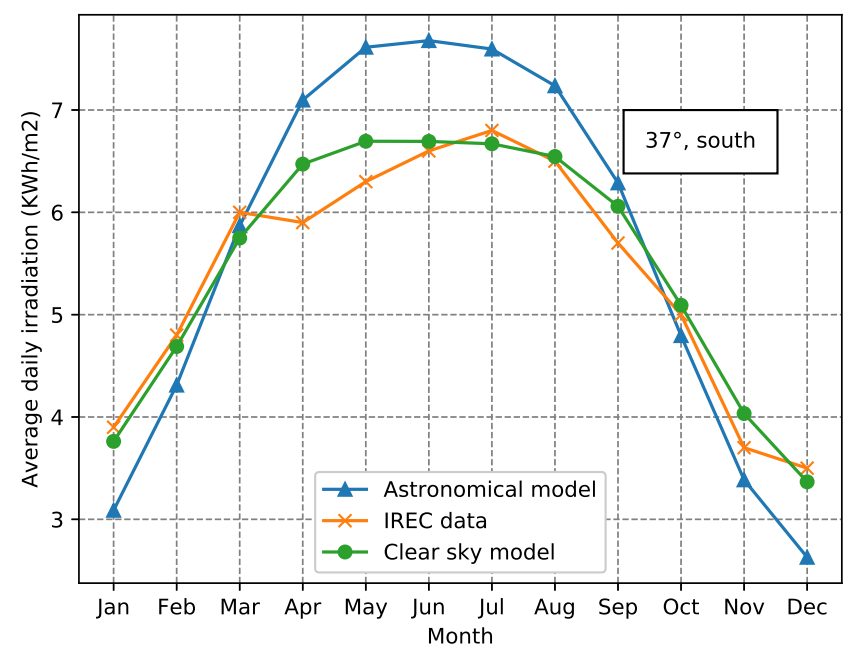

FIGURE 3 Comparison of average daily irradiation for $37^{\circ}$

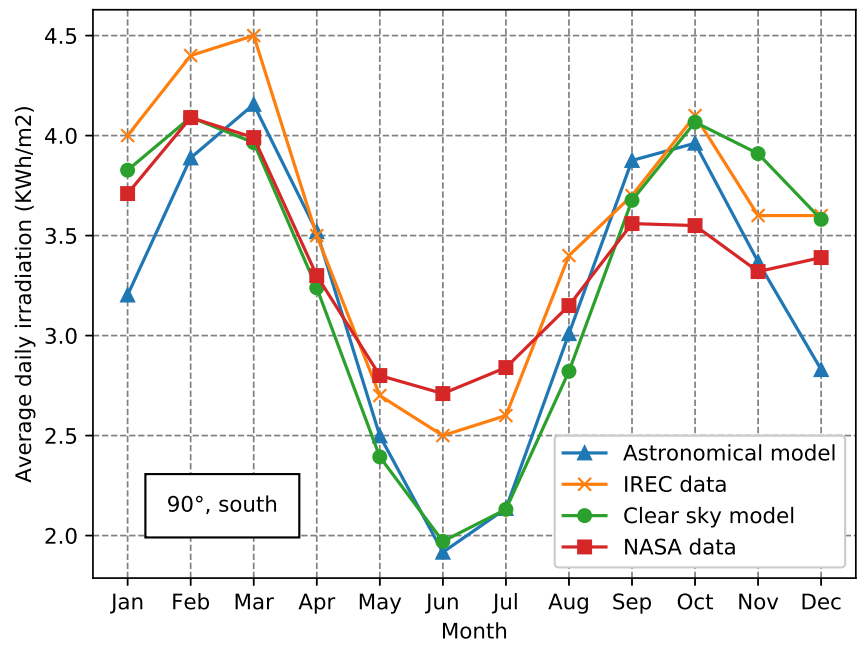

FIGURE 4 Comparison of average daily irradiation for $90^{\circ}$

\section{2 | PV power output modeling}

In general, the power output depends on the active area of the solar panel and the technology [21]:

$$
E=A_{p v} \cdot r \cdot G_{T} \cdot P R,
$$


where $A$ is the total solar panel area $\left(m^{2}\right), r$ is a solar panel yield of efficiency $(\%), G_{T}$ is an annual average solar radiation on a tilted panel (shading is not included) that depends to solar position, cloud cover, atmospheric transmittance, and power orientation; moreover, $P R$ is a performance ratio, i.e. a corrective coefficient for losses (in the range between 0.5 and 0.9 , with a default value of 0.75 ), and finally $r$ is the effective power, derived from standard test conditions (STC), that corresponds to $1000 \mathrm{~W} / \mathrm{m}^{2}$, at a cell temperature of $25^{\circ} \mathrm{C}$, wind speed $1 \mathrm{~m} / \mathrm{s}, A M=1.5$.

Alternatively, solar power output depends to global solar irradiation, area of the solar panel, efficiency of the solar panel, average losses, and temperature, as per [22]:

$$
P_{p v}=\eta \cdot A_{p v} \cdot G_{T} \cdot\left[1-0.005\left(T_{c}-25\right)\right]
$$

where $\eta$ is the photoelectric conversion efficiency $(\%), T_{c}$ is the panel operation temperature $\left({ }^{\circ} \mathrm{C}\right)$. Temperature of the cell can be obtained from the following equation: $T_{\text {air }}[i]+0.035 * G_{T}[i]$, where $T_{\text {air }}$ is an hourly temperature [23].

In practice, a correct definition of $G_{T}$ is required to obtain a proper estimate of the AC power output. Alternative power output formula does not take into account the temperature, which leads to ignoring the effect of the temperature raising on the effectiveness of the solar panel.

One of the main correction factors for the solar panel output model is power losses. In particular, the main parameter derived from the clear-sky model is a global solar radiation $\left[\mathrm{W} / \mathrm{m}^{2}\right]$. The value of this parameter significantly changes according to the meteorological factors, shading etc., and moreover, other losses occur in the solar panel itself. In general, other loss parameters can be included, for example: annual losses due to the soil, inverter losses, DC cable losses, AC cable losses, shading, losses at weak radiation, losses due to the dust, snow, and so on [24].

\subsection{State of the charge modeling}

SoC can be defined as a rate of available capacity (in Ah) against its nominal capacity [25]. In the literature, we can find common methods to estimate SoC, however these methods are just general representation and lack many details, as they usually do not consider a realistic battery behavior, but rather define SoC based on energy consumption, arrivals of energy, and battery capacity.

In addition, complex calculations and high computational cost are other concerns that make the estimation process very difficult. Exhaustive classification of SoC estimation methods are presented in [26] and [27]. Few general SoC definitions are presented below.

SoC can be defined as a relation between current capacity $(Q(t))$ and nominal capacity $\left(Q_{n}\right): \operatorname{SoC}(t)=\frac{Q(t)}{Q_{n}}[27]$.

The most common way to estimate $S o C$ is current integration: $S o C=1-\frac{\int i d t}{C_{n}}$, where $i$ is a battery current and $C_{n}$ is a nominal capacity.

Another common way to define $S o C$ is through Coulomb efficiency: $S o C=1-\frac{\int \eta i d t}{C_{n}}$, where $i$ is a positive/negative current, $\eta$ is Coulomb efficiency, i.e. the ratio of the energy required for charging to the discharging energy needed to regain the original capacity. This method requires the knowledge of initial So $C$ and precise measurements of the battery current. Coulomb method is not precise and does not include duty cycle and temperature. Apart from it, additional equipment is necessary for SoC calculation.

Another general model for defining SoC of a battery was presented in [28]: 


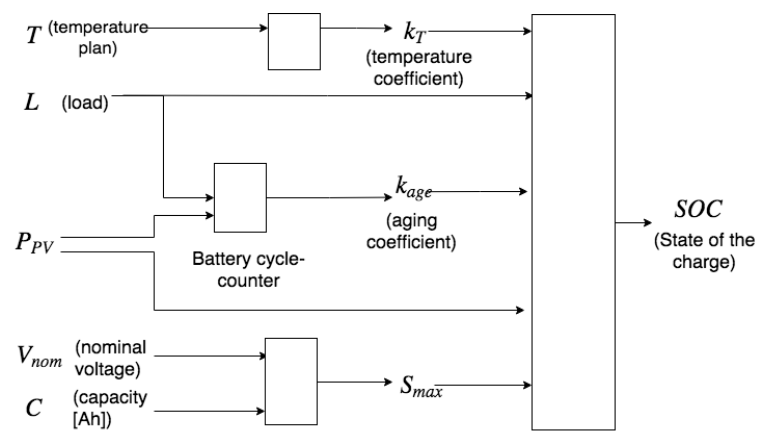

FIGURE 5 SoC estimation model

$$
S o C_{t}=\frac{S_{t-1}+\Delta S_{t}}{S_{\max }}
$$

$$
\Delta S_{t}=\Delta C_{t}-\Delta D_{t}-\Delta L_{t}
$$

where $\Delta C_{t}$ is the charging energy, $\Delta D_{t}$ is a demand parameter and $\Delta L_{t}$ is energy losses.

Demand $\Delta D_{t}$ is defined as:

$$
\Delta D_{t}=\Delta t P_{e, t}=\Delta t \cdot I_{t} \cdot U_{d c, t},
$$

where $P_{e, t}$ is the electric power consumption, $I_{t}$ is the discharging current, $U_{d c, t}$ is a voltage output of the battery.

$S_{\max }$ is defined as follows:

$$
S_{\max }=C \cdot U_{n}=\Delta P_{c, t} \cdot t
$$

where $U_{n}$ is the nominal voltage, $P_{c, t}$ is the charging power at time $t$.

Due to the non-linear time-varying characteristics and electrochemical reactions, battery SoC cannot be defined directly. Furthermore, the performance of the battery is highly affected by aging, temperature variation, chargedischarge cycle, which make the task of accurately estimating the SoC very challenging. We consider an SoC model based on the reasonings above, but we should also include additional parameters such as battery age and temperature coefficient. The general scheme of the model is reported in Fig. 5.

The current SoC depends on the SoC on the previous time interval, capacity, and nominal voltage of the energy storage, degradation of the battery, and energy charges and consumption of the device. 


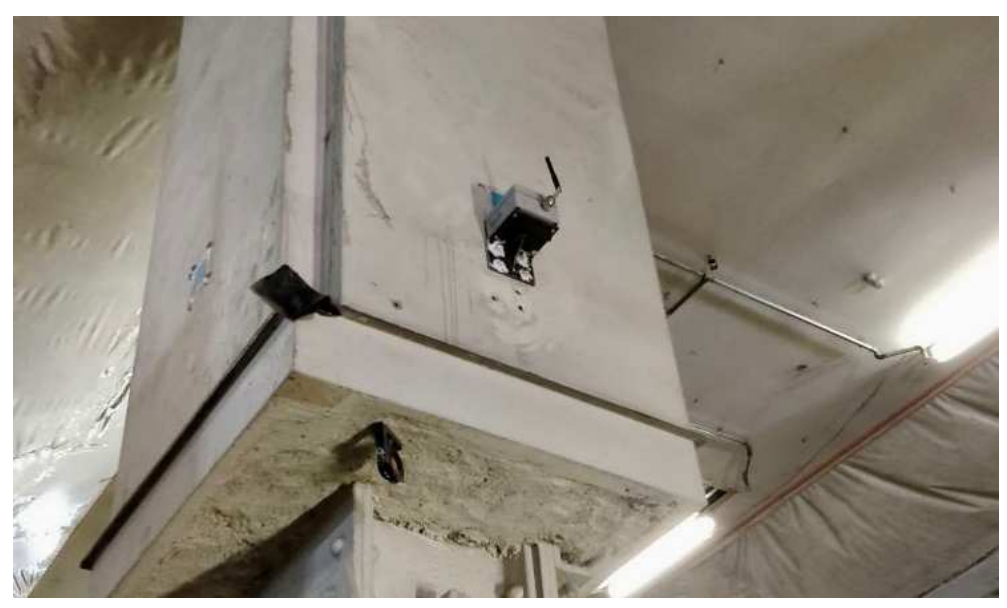

FIGURE 6 Example of tiltmeter installation for Courtesy of Sixense Oceania

\section{5 | NUMERICAL RESULTS}

In this section, we report the numerical experiments we conducted to compare different sampling strategies: constant sampling rate, DDASA with and without limits and methods, presented in Section 3.

All algorithms were tested on the tiltmeter data extracted from LS, that is a part of the Auckland City Rail Link Extension project [29]. Tiltmeters can be used to measure the surfaces' inclination of construction objects. An example of LS tiltmeter installation is presented in Fig. 6. Replacing the batteries in such objects is problematic and not economically profitable. Powering of tiltmeters by solar panels can be considered as a valid solution for the outdoor construction objects (bridges, buildings etc.).

\section{1 | LS description and energy consumption}

LS is a wireless data logger powered by batteries. It performs periodic sensing and sends the measures via radio transmission to a gateway or concentrator. It has multiple possible configurations, which affect the battery life drastically. LS can be configured to employ different duty cycles of measurements, from one measure every 30 seconds to one measure per day. The product is designed for the geotechnical industry and usually installed at locations that are difficult to reach, therefore where battery replacement to be avoided. In order to create an accurate estimation model of the battery discharge, it is necessary to outline the application scenario. In this paper, we consider worst case energy consumption scenario, determined by:

1. warming up - 3 seconds $(60 \mathrm{~mA}, 12 \mathrm{~V})$

2. measurement -3 seconds $(60 \mathrm{~mA}, 12 \mathrm{~V})$

3. transmission - 3 pulses ( $900 \mathrm{~ms}, 120 \mathrm{~mA}, 3.6 \mathrm{~V}$ each) and time between pulses ( $2 \mathrm{~s}, 15 \mathrm{~mA}, 3.6 \mathrm{~V})$

4. background consumption between cycles is $30 \mu \mathrm{Ah}, 3.6 \mathrm{~V}$.

After a measure is taken, it is sent by radio. The system has about 5 minutes to send the radio message. The message transmission has also multiple variables but for the sake of simplification we consider the worst case. 


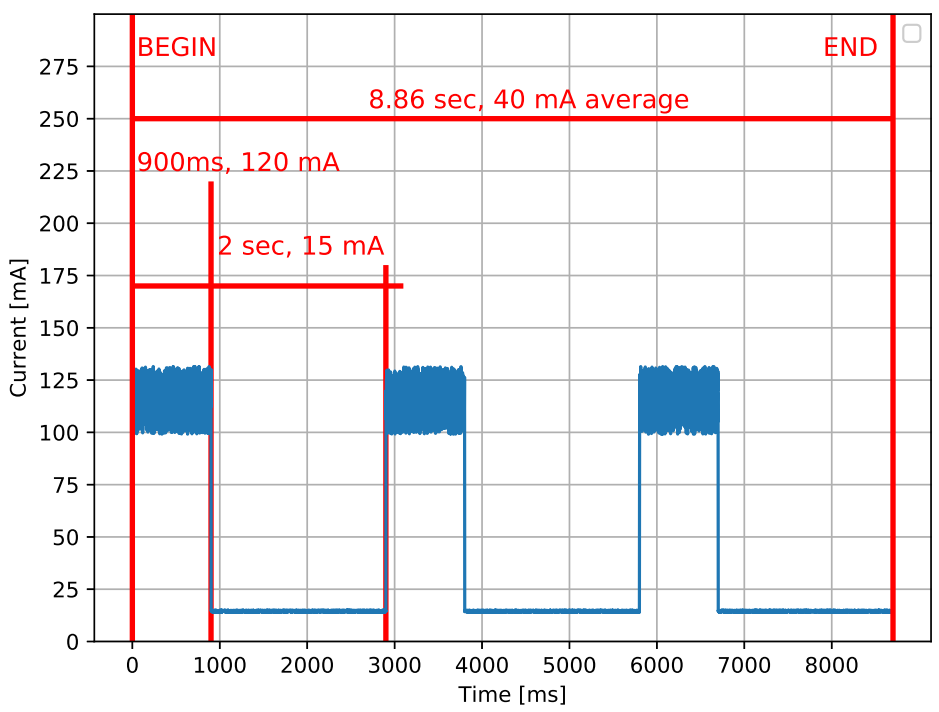

FIGURE 7 Radio consumption profile of LS device

When LS performs a complete cycle once per hour, then the hourly consumption is about $2.844 \mathrm{~W}$. The radio transmission consumption is presented in Fig. 7.

\section{2 | Solar panel characteristics}

We obtained the power output for the solar panel SOLEM 10/150/100 TD with size $138.8 \mathrm{~mm} \times 90 \mathrm{~mm}$ oriented on the south with inclination $37^{\circ}$. The theoretical efficiency of the amorphous silicon PV module is $12.7 \%$, plus average losses due to the shading, dust, wiring etc. are included with a loss coefficient of 0.75 , see Fig. 8.

The temperature dataset for solar panel power output estimation (Fig. 9) is extracted for Barcelona, Spain for 01.01.2017 - 31.12.2017 from [20].

\section{3 | Battery characteristics}

The battery present in the simulator is an LG18650B4 with nominal capacity of $2600 \mathrm{mAh}$ and nominal voltage of $3.6 \mathrm{~V}$.

The coefficient of aging was obtained from data, provided by IREC. The capacity of the battery depends on the number of cycles performed: after 300 cycles the battery loses capacity from 2600 to about 2500 mAh. In addition, battery capacity depends on the air temperature and varies from $59 \%$ of total capacity if the air temperature is below $-20^{\circ} \mathrm{C}$ to $104 \%$ if the temperature exceeds $40^{\circ} \mathrm{C}$.

\subsection{Evaluation of simulation results}

The proposed algorithms are aimed to balance irregular energy arrivals. In line with this, we set the benchmark case, that corresponds to the ideal scenario of regular energy arrivals. To do so, we averaged the energy arrival profile presented 


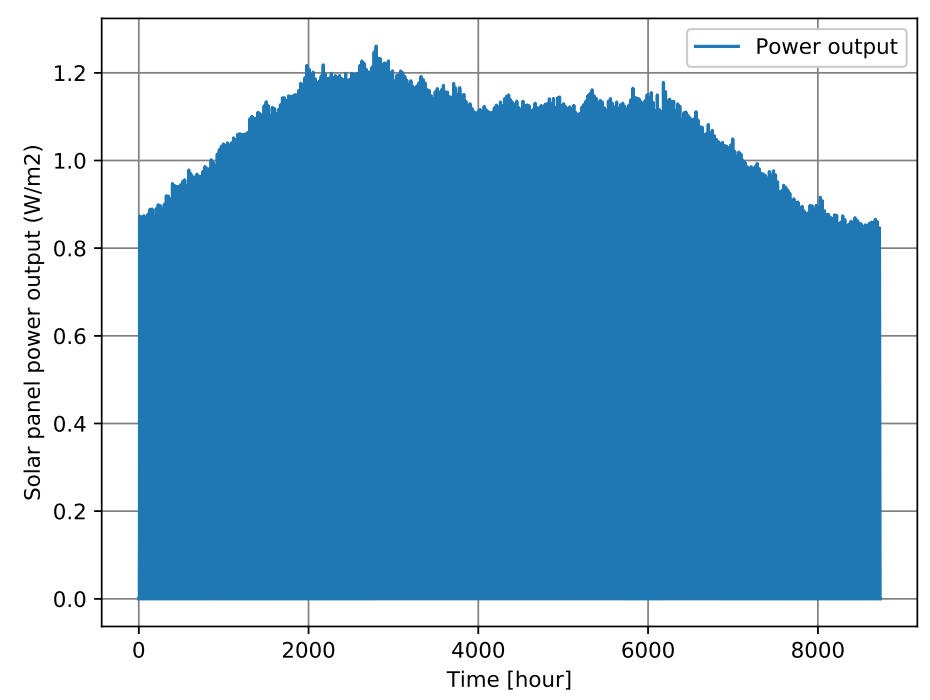

FIGURE 8 Theoretical power output of solar panel SOLEM 10/150/100 (south, 37\%) located in Barcelona, Spain throughout a year.

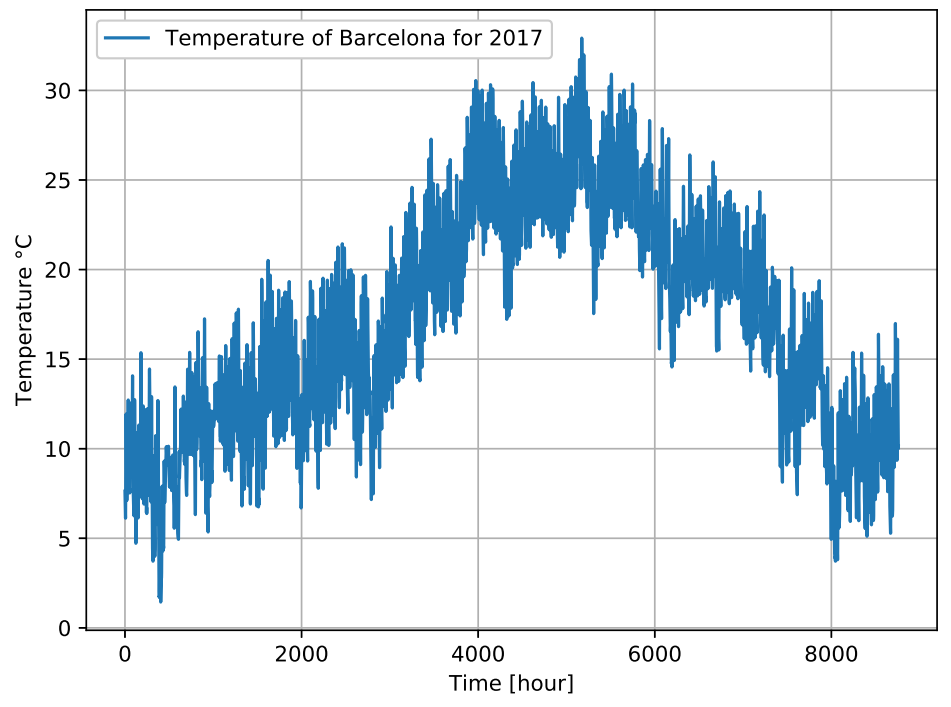

FIGURE 9 Hourly air temperature profile of Barcelona, Spain for 2017 year

in Fig. 10(a) over time.

We compare the performance of DDASA and all other proposed algorithms with the sensor performance under ideal conditions. Simulation settings are presented in Table 2 . The duration of a time slot is one hour. 


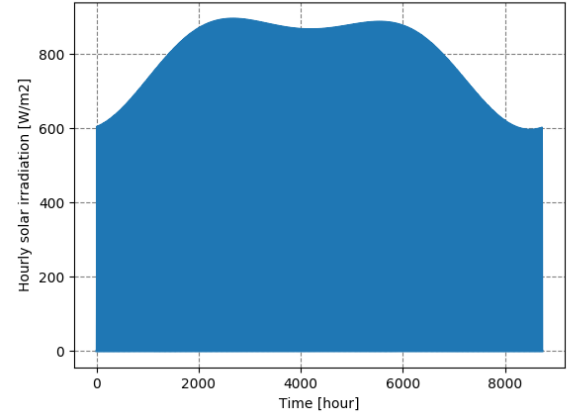

(a) realistic solar irradiation profile for Barcelona, 2017

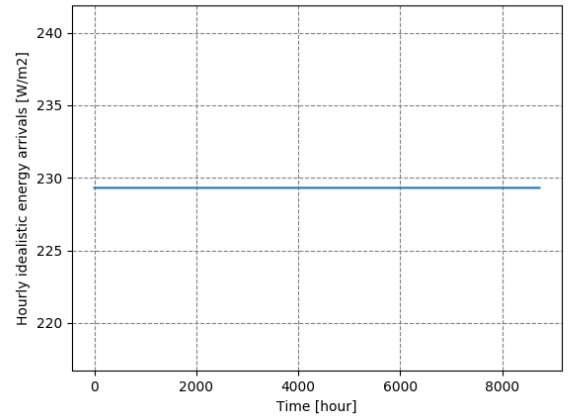

(b) Idealistic energy arrivals

FIGURE 10 Comparison of energy arrival profiles.

TABLE 2 Simulation parameters

\begin{tabular}{|l|c|}
\hline Parameter & Value \\
\hline Minimal sampling rate, $F_{\min }$ & $1.157 \cdot 10^{-5} \mathrm{~Hz}$ (24h) \\
\hline Maximum sampling rate, $F_{\max }$ & $4.639 \cdot 10^{-5} \mathrm{~Hz}$ (6h) \\
\hline Algorithm parameter, $m$ & 1 \\
\hline Algorithm parameter, $N$ & 0.5 \\
\hline Initial number of samples, $N_{D D A S A}$ & 50 \\
\hline Inclination data threshold, $t$ & 0.001 \\
\hline Harvested energy threshold, $k$ & 0.1 \\
\hline Battery level threshold, $E_{t h}$ & $0.2 S o C$ \\
\hline Upper battery level threshold & $0.4 S o C$ \\
\hline Lower battery level threshold & 0.1 SoC \\
\hline Coefficient, $\alpha$ & 1.2 \\
\hline Coefficient, $\beta$ & 0.8 \\
\hline
\end{tabular}

The failure rate is chosen as a comparison performance metric. A device fails when the battery does not have enough energy to transmit a data packet. If the significant gain in decreasing of failure rate by adapting the algorithm is achieved, then we will have simple and effective lightweight solution, which can be implemented on the real sensor devices.

The implementation of DDASA algorithm in LS demonstrated that the algorithm needs to be improved in terms of energy awareness and robustness, that have to be more balanced and adapted to the available energy level and harvesting capabilities. 


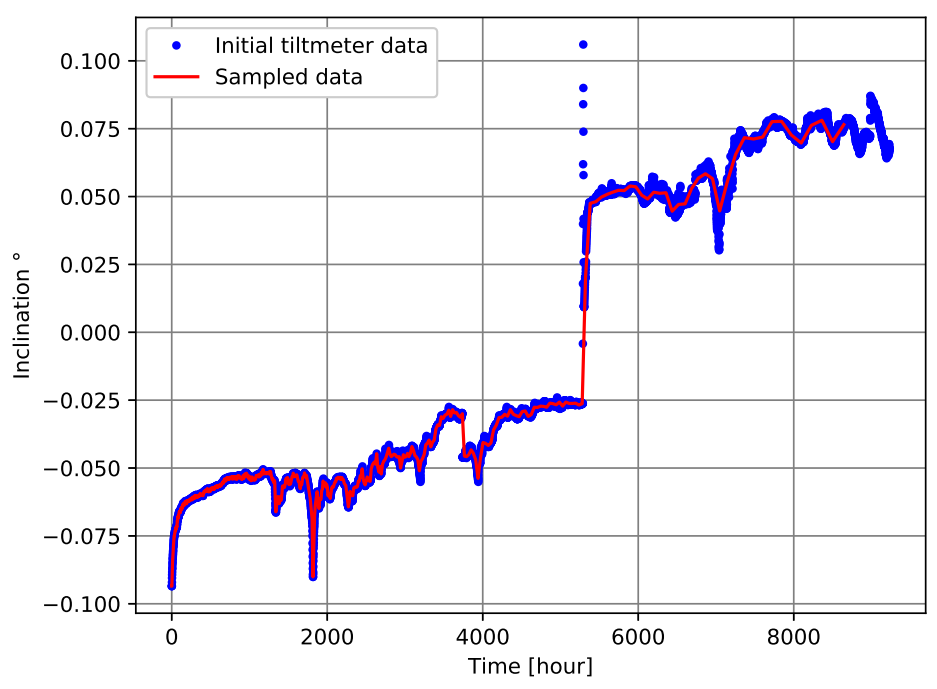

FIGURE 11 Comparison of initial dataset obtained under constant sampling rate of 1 hour with a dataset obtained under DDASA (here initial tiltmeter data and sampled data respectively)

DDASA leads LS device to frequent failures to transmit a data packet (Table 3). Sampling rate obtained with DDASA depends to the data variation only. And it can lead to the situation when the energy arrivals are poor, but data variation is high. It causes more aggressive battery drain and termination of LS operation. Therefore, the original version of DDASA is not able to ensure the robust operation of LS that is powered by a solar panel over a whole year.

The sampling rate during data collection phase (or transition phase) is adjusted to 1 hour, which is the duration of a time slot. All device failures of the proposed strategies (T-ASA, L-ASA, LT-DDASA, EA-DDASA) are accounted for this transition period. If we compare the similar throughput results presented in Table 2 , then EA-DDASA provides the closest performance results to the ideal conditions case. The failure rate is 0 during all months except January, that includes the transition phase 12(a). EA-DDASA demonstrates balanced energy consumption 12(b): during winter it consumes less energy, while during summer months it consumes more energy, except July, that can be explained by the power output pattern, shown in Fig. 8. DDASA energy consumption is guided by data variations and therefore the energy consumption is unbalanced, and during some winter months we observe much higher energy consumption, than during summer months, which causes the device to operate on the edge of its capabilities.

In general, the choice of the algorithm can be dictated by different circumstances. In particular, if the environmental conditions have a stable pattern over the span of the year (i.e., energy provision has little volatility), then L-ASA can be adopted, since it provides a higher throughput, but the average SoC is lower, comparing to other proposed algorithms. If the environmental characteristics are highly unstable, then T-ASA can be implemented, that provides the highest average SoC. The most balanced methods are LT-DDASA and EA-DDASA. In addition, if the Li-ion battery is attached to the device, then the recommended energy level holds. For some batteries chemistry, it is preferable to keep the average battery level low to preserve the battery life [30]. As the battery level stays around $100 \%$ SoC, the battery degrades faster, since Keeping charging the battery leads to micro-charges and discharges, thus negatively affecting the battery's life. Therefore, the average SoC may be also worth considering.

In order to improve robustness of the proposed schemes, the energy arrivals learning models can be implemented, 
TABLE 3 Comparison of algorithms

\begin{tabular}{|l|c|c|c|c|}
\hline \multicolumn{1}{|c|}{ Algorithm } & $\begin{array}{c}\text { Throughput } \\
\text { [packets] }\end{array}$ & $\begin{array}{c}\text { Failure rate (with/ } \\
\text { without transition } \\
\text { phase) }\end{array}$ & $\begin{array}{c}\text { Total energy } \\
\text { consumption, } \\
{[\mathbf{W}]}\end{array}$ & $\begin{array}{c}\text { Average } \\
\text { SoC, } \%\end{array}$ \\
\hline Constant energy arrivals, sampling rate (24h) & 364 & 0.00 & 1036 & 99.5 \\
\hline Constant energy arrivals, sampling rate (9h) & 970 & 0.00 & 2759 & 98.1 \\
\hline Constant energy arrivals, sampling rate (6h) & 1028 & 0.05 & 2919 & 5.8 \\
\hline Realistic energy arrivals, sampling rate (24h) & 364 & 0.00 & 1036 & 98.5 \\
\hline Realistic energy arrivals, sampling rate (6h) & 938 & 0.06 & 2668 & 5.3 \\
\hline DDASA & 1336 & $0.58 / 0.56$ & 7603 & 3.0 \\
\hline DDASA with limits & 951 & $0.04 / 0.02$ & 5414 & 5.6 \\
\hline T-ASA & 407 & $0.02 / 0.00$ & 2319 & 92.8 \\
\hline L-ASA & 945 & $0.02 / 0.00$ & 5379 & 19.9 \\
\hline LT-DDASA & 709 & $0.02 / 0.00$ & 4037 & 75.7 \\
\hline EA-DDASA & 915 & $0.02 / 0.00$ & 5209 & 68.9 \\
\hline
\end{tabular}

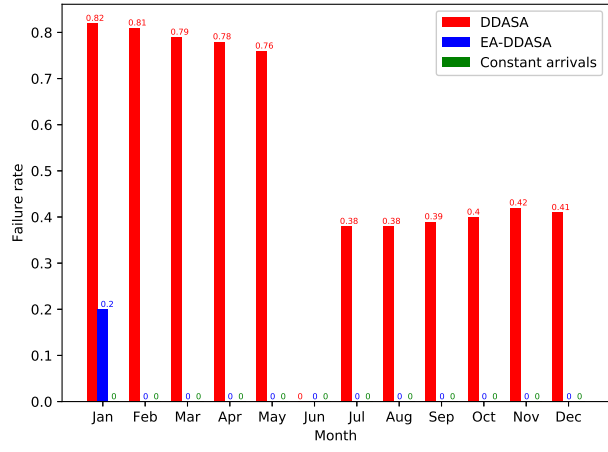

(a) Monthly failure rate

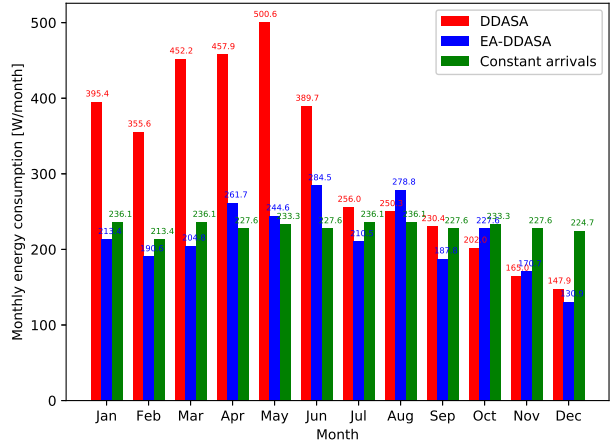

(b) Monthly energy consumption

FIGURE 12 Comparison of DDASA, ideal conditions strategy and EA-DDASA.

that will exclude the usage of the predefined environmental characteristic evaluations. This method is more effective, but at the same time computationally heavy and requires to install additional hardware, that measures the solar radiation information (pyranometers). This will lead to the overall cost increase of a sampling device. 


\section{6 | CONCLUSIONS}

One of the effective way to provide the robustness to a sensor node operation is to adapt the sampling rate. The efficient adaptive sampling strategy adopted in an energy harvesting sensor node is required to provide a failsafe operation under unstable environmental conditions, and be implementable in the existing hardware. In line with this, we proposed energy aware strategies applied to the data driven adaptive sampling approach, that balance the energy consumption and decrease the number of packet delivery failures. To validate the performance of the proposed schemes, we simulated the operation of the industrial data-logger powered with a solar panel located in Barcelona, Spain.

We observed that with prior knowledge of the environmental characteristics it is reasonable to set threshold based rules and sampling rate limits that significantly increase the performance of the existing data-driven approach without increasing the complexity of the algorithm.

Improving sensor operation strategies is needed to provide the full autonomy of a device with energy harvesting capabilities, which is a key to design successful and self-sustainable loT systems.

\section{Appendices}

\section{A | SOLAR IRRADIATION MODELS}

The following groups of solar irradiation models can be outlined: linear and non-linear. Linear models give the correlation between solar energy on a horizontal surface and some meteorological variables, such as shining hours, ambient temperature and relative humidity. Due to the model simplicity, linear models are more commonly used. Diffuse solar energy models describe the relationship between the average daily diffuse and global solar radiations incident on a horizontal surface and the sky clearness index.

Other more sophisticated types of models are based on the artificial neural networks (ANN). The commonly used input variables in ANN-based models are the sunshine ratio, ambient temperature, and relative humidity to predict global solar energy at different locations, but also following inputs can be used: latitude, longitude, altitude, month, time, wind speed, relative humidity, and rainfall. The results of the study showed that the ANN-based models are more accurate in predicting the diffuse radiation compared to the linear regression models, but are much more demanding in terms of data and complexity.

In [31], the astronomical solar model is presented, which is used to translate the instantaneous solar radiation $\left(I_{\text {sun }}\right)$ into effective radiation. The effective (or available) solar radiation $\left(I_{\text {eff }}=I_{\text {sun }} \cdot \cos \Theta\right.$ ) is dependent on factors such as: location, inclination of a solar module, time of the year and hour of the day, where $\Theta$ is the angle between the sunlight and the normal to the solar module surface.

In [32], a clear-sky radiation model is introduced. The total radiation $G_{T}$ is divided on 3 components: beam $\left(G_{b T}\right)$,diffuse $\left(G_{d T}\right)$, and reflection $\left(G_{r T}\right)$, which can be calculated as:

$$
\begin{gathered}
G_{b T}=G_{o n} \tau_{b} \cos \theta_{s} \\
G_{d T}=G_{o n} \cos \theta_{z} \tau_{d}\left(\frac{1+\cos \beta}{2}\right) \\
G_{r T}=\rho G_{o n} \cos \theta_{z} \tau_{r}\left(\frac{1+\cos \beta}{2}\right)
\end{gathered}
$$


where $G_{o n}$ is the solar radiation outside of the atmosphere, $\tau_{b}, \tau_{d}$ and $\tau_{r}$ are the atmospheric transmittance for a beam, diffuse and reflected solar radiation, respectively. $\theta_{z}, \theta_{s}, \beta$ and $\rho$ are the solar zenith angle ( $\mathrm{rad}$ ), the incident angle on the surface, the inclination angle of the surface $(\mathrm{deg})$ and the average reflection on the ground.

The clear-sky model is suitable for meteorological conditions without clouds, mist or haze, but in comparison with the astronomical model, it includes the diffusion and reflection components.

Astronomical and clear-sky models do not include atmospheric attenuation and are not as accurate as ANN models. However, these models do not require meteorological data and solar radiation measurements, therefore the model is easily applicable and can be adapted to any location. Clear-sky solar radiation model is a wider model that includes parameters such as diffusion and reflection solar energy. Therefore, this model can be used as a foundation to compute the solar radiation in a particular location for a solar panel with known inclination angle and direction.

\section{REFERENCES}

[1] Green MA, Hishikawa Y, Warta W, Dunlop ED, Levi DH, Hohl-Ebinger J, et al. Solar cell efficiency tables (version 50). Progress in Photovoltaics: Research and Applications 2017;25(7):668-676.

[2] Shu T, Xia M, Chen J, de Silva C. An Energy Efficient Adaptive Sampling Algorithm in a Sensor Network for Automated Water Quality Monitoring. Sensors 2017;17(11):2551.

[3] Worldsensing | Loadsensing | Mining and Construction Monitoring;. https://www.worldsensing.com/product/ loadsensing/.

[4] Khan JA, Qureshi HK, Iqbal A. Energy management in wireless sensor networks: A survey. Computers \& Electrical Engineering 2015;41:159-176.

[5] Kansal A, Hsu J, Zahedi S, Srivastava MB. Power management in energy harvesting sensor networks. ACM Transactions on Embedded Computing Systems (TECS) 2007;6(4):32.

[6] Polastre J, Szewczyk R, Culler D. Telos: enabling ultra-low power wireless research. In: Proceedings of the 4th international symposium on Information processing in sensor networks IEEE Press; 2005. p. 48.

[7] Juang P, Oki H, Wang Y, Martonosi M, Peh LS, Rubenstein D. Energy-efficient computing for wildlife tracking: Design tradeoffs and early experiences with ZebraNet. ACM SIGARCH Computer Architecture News 2002;30(5):96-107.

[8] Alippi C, Anastasi G, Di Francesco M, Roveri M. Energy management in wireless sensor networks with energy-hungry sensors. IEEE Instrumentation \& Measurement Magazine 2009;12(2).

[9] Alippi C, Anastasi G, Di Francesco M, Roveri M. An adaptive sampling algorithm for effective energy management in wireless sensor networks with energy-hungry sensors. IEEE Transactions on Instrumentation and Measurement 2010;59(2):335-344.

[10] Srbinovski B, Magno M, Edwards-Murphy F, Pakrashi V, Popovici E. An energy aware adaptive sampling algorithm for energy harvesting WSN with energy hungry sensors. Sensors 2016;16(4):448.

[11] Lee C, Lee J. Harvesting and Energy aware Adaptive Sampling Algorithm for guaranteeing self-sustainability in Wireless Sensor Networks. In: Information Networking (ICOIN), 2017 International Conference on IEEE; 2017. p. 57-62.

[12] Yang J, Wu X, Wu J. Adaptive sensing scheduling for energy harvesting sensors with finite battery. In: Communications (ICC), 2015 IEEE International Conference on IEEE; 2015. p. 98-103.

[13] Nguyen LV, Kodagoda S, Ranasinghe R, Dissanayake G. Information-driven adaptive sampling strategy for mobile robotic wireless sensor network. IEEE Transactions on Control Systems Technology 2016;24(1):372-379. 
[14] Xu Y, Choi J, Dass S, Maiti T. Sequential Bayesian prediction and adaptive sampling algorithms for mobile sensor networks. IEEE Transactions on Automatic Control 2012;57(8):2078-2084.

[15] Rieger R, Taylor JT. An adaptive sampling system for sensor nodes in body area networks. IEEE Transactions on Neural Systems and Rehabilitation Engineering 2009;17(2):183-189.

[16] Sun Y, Yuan Y, Li X, Xu Q, Guan X. An Adaptive Sampling Algorithm for Target Tracking in Underwater Wireless Sensor Networks. IEEE Access 2018;6:68324-68336.

[17] Yin X, Goudriaan J, Lantinga EA, Vos J, Spiertz HJ. A flexible sigmoid function of determinate growth. Annals of botany 2003;91(3):361-371.

[18] Hofierka J, Suri M, et al. The solar radiation model for Open source GIS: implementation and applications. In: Proceedings of the Open source GIS-GRASS users conference, vol. 2002; 2002. p. 51-70.

[19] Khatib T, Mohamed A, Sopian K. A review of solar energy modeling techniques. Renewable and Sustainable Energy Reviews 2012;16(5):2864-2869.

[20] SoDA: Solar radiation data;. [Online; accessed 09-May-2018]. http://www. soda-pro.com/.

[21] Chong W, Naghavi M, Poh S, Mahlia T, Pan K. Techno-economic analysis of a wind-solar hybrid renewable energy system with rainwater collection feature for urban high-rise application. Applied Energy 2011;88(11):4067-4077.

[22] Akram U, Khalid M, Shafiq S. An Innovative Hybrid Wind-Solar and Battery-Supercapacitor Microgrid System-Development and Optimization. IEEE Access 2017;5:25897-25912.

[23] Migan GA. Study the operating temperature of a PV module. Project Report 2013;.

[24] Maghami MR, Hizam H, Gomes C, Radzi MA, Rezadad MI, Hajighorbani S. Power loss due to soiling on solar panel: A review. Renewable and Sustainable Energy Reviews 2016;59:1307-1316.

[25] Murnane M, Ghazel A. A Closer Look at State of Charge (SOC) and State of Health (SOH) Estimation Techniques for Batteries. Analog Devices Technical Article, $<$ http://www analog com/media/en/technicaldocumentation/technicalarticles/A-Closer-Look-at-State-Of-Charge-and-State-Health-Estimation-Techniques- pdf 2017;

[26] Hannan MA, Lipu M, Hussain A, Mohamed A. A review of lithium-ion battery state of charge estimation and management system in electric vehicle applications: Challenges and recommendations. Renewable and Sustainable Energy Reviews 2017;78:834-854.

[27] Chang WY. The state of charge estimating methods for battery: A review. ISRN Applied Mathematics 2013;2013.

[28] Homan B, Smit GJ, van Leeuwen RP, Marnix V, Ten B. A comprehensive model for battery State of Charge prediction. In: PowerTech, 2017 IEEE Manchester IEEE; 2017. p. 1-6.

[29] City Rail Link;. https://www.cityraillink.co.nz/.

[30] Millner A. Modeling lithium ion battery degradation in electric vehicles. In: 2010 IEEE Conference on Innovative Technologies for an Efficient and Reliable Electricity Supply IEEE; 2010. p. 349-356.

[31] Miozzo M, Zordan D, Dini P, Rossi M. SolarStat: Modeling photovoltaic sources through stochastic Markov processes. In: Energy Conference (ENERGYCON), 2014 IEEE International IEEE; 2014. p. 688-695.

[32] Tao C, Shanxu D, Changsong C. Forecasting power output for grid-connected photovoltaic power system without using solar radiation measurement. In: Power Electronics for Distributed Generation Systems (PEDG), 2010 2nd IEEE International Symposium on IEEE; 2010. p. 773-777. 


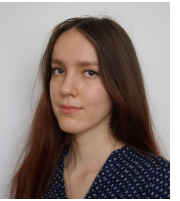

ELVINA GINDULLINA received her M.S. degree (with honor) in applied mathematic and computer science from Ufa State Aviation technical University (USATU), Russia, in 2015. From 2014 to 2016 she joined USATU as a programmer. She is currently pursuing her Ph.D. in University of Padova (Italy) in the Department of Information Engineering. In September 2016 she joined SCAVENGE project as a ESR, under the Marie Skłodowska-Curie Actions programme. During January - July 2018 she conducted her internship in Worldsensing (Barcelona, Spain) as a research engineer. Her research interests include battery management systems, energy cooperation solutions and increasing of energy efficiency for energy harvesting loT networks.

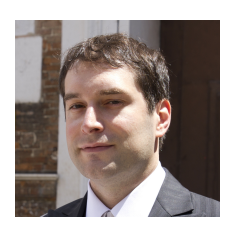

LEONARDO BADIA (S'02-M'04-SM'13) received the Laurea degree (with honors) in electrical engineering and the PhD degree in information engineering from the University of Ferrara, Italy, in 2000 and 2004, respectively. During 2002 and 2003, he was on leave at the Radio System Technology Labs (now Wireless@KTH), Royal Institute of Technology of Stockholm, Sweden. After having been with the Engineering Department of the University of Ferrara, he joined in 2006 the IMT Institute for Advanced Studies, in Lucca, Italy. In 2011, he moved to the University of Padova, Italy, where he is currently an Associate Professor. His research interests include protocol design for multi-hop networks, cross-layer optimization of wireless communication, transmission protocol modeling, and applications of game theory to radio resource management. Professor Badia published more than 140 research papers. He is involved in the coordination of scientific events and projects. He is also an active referee of research articles, having served on the editorial boards and still being an active reviewer for many scientific periodicals, as well as technical program committee chair for conferences in the broad area of networking and wireless communications.

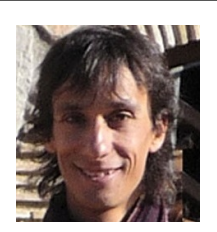

XAVIER VILAJOSANA is a computer science engineer, co-founder of Worldsensing and OpenMote Technologies. He is currently Principal Investigator at the Wireless Networks Research Lab at the Universitat Oberta de Catalunya. From January 2012 to January 2014, Xavier was visiting Professor at the University of California Berkeley holding a prestigious Fulbright fellowship. In 2008, he was visiting researcher of France Telecom R\&D Labs, Paris. Xavier has been one of the main promoters of low power wireless technologies, co-leading the OpenWSN.org initiative at UC Berkeley, and promoting the use of low power wireless standards for the emerging Industrial Internet paradigm. He also contributed to the industrialization and introduction of Low Power Wide Area Networks to urban scenarios through Worldsensing. Xavier is author of different Internet Drafts, as part of his standardization activities for low power industrial networks. Xavier is contributing actively at the IETF 6TiSCH WG. He holds an MsC degree on Computer Science from the Universitat Politècnica de Catalunya (UPC) and a PhD on Computer Science from the Universitat Oberta de Catalunya. At the moment, Xavier is author of 30 patents, more than 40 high impact journal publications and more than 50 International conference contributions. Technically, Xavier has extensive experience in Distributed Systems, Wireless Networks and Industrial Networks. 